\title{
Aortic regurgitation caused by rupture of a well-balanced fibrous strand suspending a degenerative tricuspid aortic valve
}

\author{
Masato Nakajima, MD, Kouji Tsuchiya, MD, Yuji Naito, MD, Narutoshi Hibino, MD, and Hidenori Inoue, MD, \\ Kofu City, Yamanashi, Japan
}

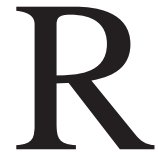

upture of the aortic valve is recognized as one of the causes of acute aortic regurgitation. ${ }^{1}$ However, rupture of a fibrous strand of a tricuspid aortic valve, not associated with bicuspid aortic valve, commissural dehiscence, fenestration, or trauma, is extremely rare. We describe the case of a patient with aortic regurgitation caused by spontaneous rupture of one of several well-balanced fibrous strands that suspended the three aortic valve leaflets.

\section{Clinical Summary}

A 52-year-old man was transferred to our hospital for surgical treatment of severe aortic regurgitation. The patient had hypertension that was controlled with medical treatment, with no history of rheumatic heart valve disease. His hemodynamic condition had deteriorated several months before admission, but there were no signs of infective endocarditis. Preoperative echocardiography and cardiac catheterization, including angiography, revealed pure aortic regurgitation.

The patient underwent elective surgery. During the operation it was discovered that each of the three leaflets had a pair of fibrous strands between it and the ascending aortic wall (Figure 1). These originated from the sinotubular junction just above each commissure and suspended the free margin of the nearest aortic valve leaflet. They were well balanced, but one of the strands, which had previously suspended the right coronary cusp, was ruptured, and

From the Department of Cardiovascular Surgery, Yamanashi Central Hospital, Kofu City, Yamanashi, Japan.

Received for publication Jan 28, 2002; accepted for publication Feb 16, 2002.

Address for reprints: Masato Nakajima, MD, Department of Cardiovascular Surgery, Yamanashi Central Hospital, 1-1-1 Fujimi, Kofu City, Yamanashi 400-0027, Japan (E-mail: m-nakajima2a@ych.pref.yamanashi.jp).

J Thorac Cardiovasc Surg 2002;124:843-4

Copyright (C) 2002 by The American Association for Thoracic Surgery

$0022-5223 / 2002 \$ 35.00+0 \quad \mathbf{1 2 / 5 4 / 1 2 4 2 9 6}$

doi:10.1067/mtc.2002.124296

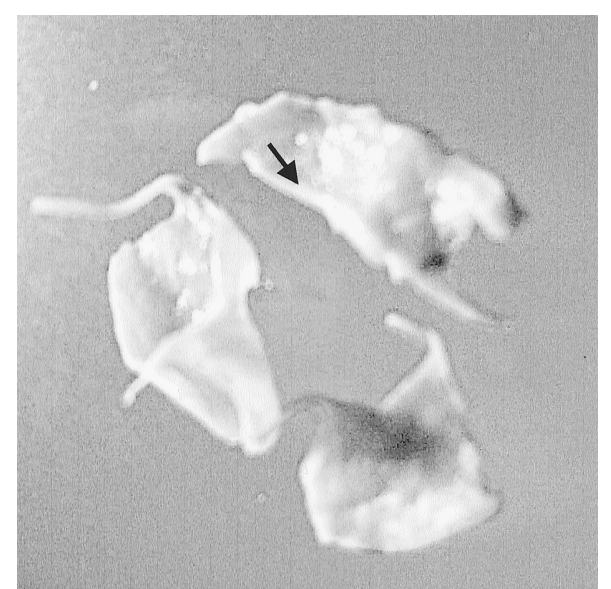

Figure 1. Resected aortic valve showing six fibrous strands, with one strand ruptured (black arrow).

the cusp dropped into the left ventricular cavity. Severe aortic insufficiency was considered to have been the consequence of this rupture (Figure 2). Because degenerative change was recognized on the valve leaflet itself, we concluded that reconstruction was unsuitable. The aortic valve and all fibrous strands were resected and replaced with a mechanical heart valve. Histologic examination showed only myxoid degeneration of valves. The postoperative course was satisfactory, and the patient was discharged without complications.

\section{Discussion}

Aortic regurgitation caused by rupture of aortic valve tissue has been previously reported in Asian countries, and especially in Japan. These cases have been associated with bicuspid aortic valve, ${ }^{2-6}$ commissural rupture, ${ }^{7,8}$ fenestration, ${ }^{9}$ and abnormal fibrous strand rupture ${ }^{10}$ or extension (kite anomaly). ${ }^{11}$ The wellbalanced aortic valvular apparatus in our case is quite rare, and this form of rupture has not previously been reported. Investigation of the clinical course in conjunction with the histologic examination 


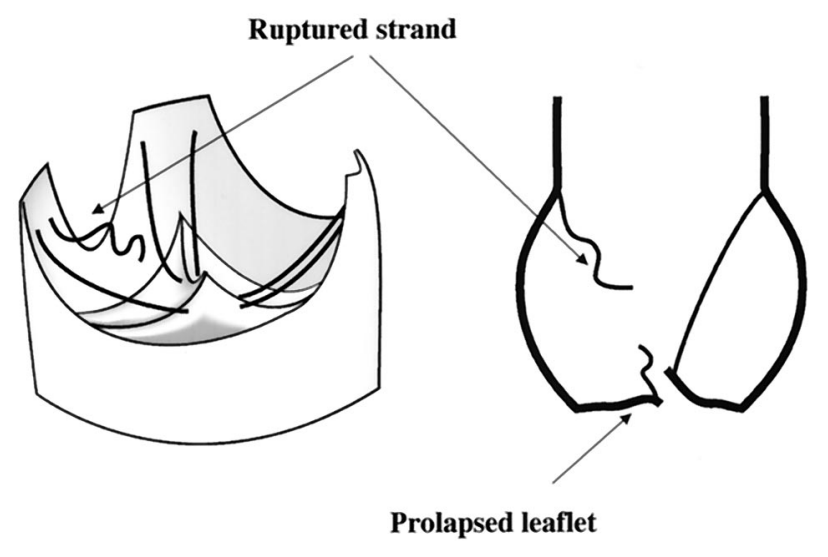

Figure 2. Mechanism of acute aortic regurgitation. Right coronary cusp was prolapsed by strand rupture.

suggests that the condition of degenerative aortic incompetence was acutely worsened by the rupture of one of the fibrous strands that had suspended an elongated leaflet.

In cases of acute aortic regurgitation other than in the presence of infective endocarditis, aortic valve rupture should be considered. Also, it is possible that there are symptom-free people with similar well-balanced aortic valve suspension systems.

From the perspective of embryology, the aortic valve and sinuses of Valsalva are formed by distal aortic bulges extending toward the ventricular cavity. So it is conceivable that this evolution would leave fibrous tissue between the aortic valve and the aortic wall. Persistence of these fibers may not normally contribute to aortic valvular function; however, in this case, the degenerative aortic valve leaflet and the six fibrous strands may have coped with the diastolic aortic pressure and maintained valve function until the rupture of a fibrous strand. Furthermore, we think that this rupture was caused by pressure load, because histologic findings revealed no inflammatory changes in the strand.
Finally, the existence of fibrous strands involved in the suspension of a degenerated aortic valve suggests to us the possibility of repair of aortic valve incompetence, especially when it is due to a degenerated and prolapsed aortic valve leaflet. Reconstruction of the three-leaflet coaptation with some kind of suspension apparatus might thus ameliorate aortic valve function in cases of prolapsing aortic regurgitation.

\section{References}

1. Becker AE, Duren DR. Spontaneous rupture of bicuspid aortic valve: an unusual cause of aortic insufficiency. Chest. 1977;72:361-2.

2. Misawa Y, Hasegawa T, Oyama H, Sudo H, Hasegawa N, Kamisawa O. [Congenital bicuspid aortic valve with regurgitation-a rare case showing a fibrous band between the conjoined cusp and the ascending aorta]. Nippon Kyobu Geka Gakkai Zasshi. 1993;41:2156-9.

3. Hamada Y, Iijima T, Yoshida I, Tsuda K, Ishikawa S, Yanagisawa H, et al. [Bicuspid aortic valve with sudden onset of aortic insufficiency due to rare causes: report of two cases]. Kyobu Geka. 1992;45:519-21.

4. Criampricotti R, Gamal ME, Mashhou YA. Acute aortic regurgitation due to spontaneous rupture of a bicuspid aortic valve: detection by echocardiography. Clin Cardiol. 1987;10:484-6.

5. Yamagishi M, Anzai N, Yamada M. An exceptional form of congenitally bicuspid aortic valve resulting in pure aortic regurgitation. Jpn Heart J. 1986;27:267-71.

6. Sugita T, Matsumoto M, Nishizawa J, et al. Aortic laceration due to prolapse of the bicuspid aortic valve: case report. $J$ Heart Valve Dis. 2001;10:375-6.

7. Satokawa H, Hoshino S, Iwaya F, Igari T, Ando M, Abe T, et al. [A case of aortic regurgitation due to spontaneous rupture of an aortic commissure]. Kyobu Geka. 1986;39:817-20.

8. Aoyagi S, Fukunaga S, Oishi K. Aortic regurgitation due to nontraumatic rupture of the aortic valve commissures: report of two cases. J Heart Valve Dis. 1995;4:99-102.

9. Akiyama K, Ohsawa S, Hirota J, Takiguchi M. Massive aortic regurgitation by spontaneous rupture of a fibrous strand in a fenestrated aortic valve. J Heart Valve Dis. 1998;7:521-3.

10. Kaneko T, Taniguchi K, Obayashi T, Murai N, Aizaki M, Tanaka T, et al. [Aortic regurgitation due to ruptured fibrous band in the noncoronary cusp: a case report]. J Cardiol. 1996;27 Suppl 2:79-82.

11. Yavuz S, Turk T, Celkan MA, Koca V, Ata Y, Ozdemir IA. Congenital aortic insufficiency due to aortic cusp stretching: 'kite anomaly'. J Heart Valve Dis. 1999;8:284-6. 